\title{
Some Hardy-Type Inequalities for Superquadratic Functions via Delta Fractional Integrals
}

\author{
Usama Hanif $\left(D^{D},{ }^{1}\right.$ Ammara Nosheen $(D),{ }^{1}$ Rabia Bibi $(D),{ }^{2}$ Khuram Ali Khan $(D), 3$ \\ and Hamid Reza Moradi ${ }^{3}{ }^{3}$ \\ ${ }^{1}$ Department of Mathematics and Statistics, The University of Lahore, Sargodha Sub-Campus, Sargodha, Pakistan \\ ${ }^{2}$ Department of Mathematics, Abbottabad University of Science and Technology, Havelian, Abbottabad, Pakistan \\ ${ }^{3}$ Department of Mathematics, University of Sargodha, Sargodha 40100, Pakistan
}

Correspondence should be addressed to Hamid Reza Moradi; hrmoradi@mshdiau.ac.ir

Received 21 March 2021; Accepted 6 May 2021; Published 28 May 2021

Academic Editor: Erhan Set

Copyright (C) 2021 Usama Hanif et al. This is an open access article distributed under the Creative Commons Attribution License, which permits unrestricted use, distribution, and reproduction in any medium, provided the original work is properly cited.

In this paper, Jensen and Hardy inequalities, including Pólya-Knopp type inequalities for superquadratic functions, are extended using Riemann-Liouville delta fractional integrals. Furthermore, some inequalities are proved by using special kernels. Particular cases of obtained inequalities give us the results on time scales calculus, fractional calculus, discrete fractional calculus, and quantum fractional calculus.

\section{Introduction}

The study of Hardy inequalities has gained huge attention in the literature, and now it became a major field in applied and pure mathematics. The Hardy inequality has a long history and many variants. Together with the Sobolev inequalities, it is one of the most frequently used inequalities in the analysis. Firstly, Hardy inequality was discovered to simplify the proof of another inequality. It was then studied in its own right and acquired several useful variants, and it eventually turned out to be extremely useful in the theory of partial differential equations. Except for the direct application of Hardy's inequality to the Schrodinger operator, other useful variants have been successfully developed for applications in other areas of physics [1].

Hardy-type inequalities have crucial importance in the study of function spaces, especially of fractional regularity [2]. Another fundamental consequence is the trace theory of weighted Sobolev spaces; in turn, weighted Sobolev spaces are useful in the regularity theory of the superposition operators [2]. The familiar Hardy inequality (both in the discrete and continuous settings) as presented in [3] has been enormously studied and used as a model for the inquisition of more general integral inequalities $[4,5]$.

In [6], Hardy proved the discrete inequality,

$$
\sum_{n=1}^{\infty}\left(\frac{1}{n} \sum_{i=1}^{n} a(i)\right)^{p} \leq\left(\frac{p}{p-1}\right)^{p} \sum_{n=1}^{\infty} a^{p}(n), \quad p>1,
$$

where $a(n) \geq 0$ for $n \geq 1$. Also, in [7], Hardy proved the continuous inequality, using the calculus of variation, which states that for nonnegative, integrable function over any finite interval $(0, x)$ and $f^{p}$ is integrable for $p>1$,

$$
\int_{0}^{\infty}\left(\frac{1}{x} \int_{0}^{x} f(t) \mathrm{d} t\right)^{p} \mathrm{~d} x \leq\left(\frac{p}{p-1}\right)^{p} \int_{0}^{\infty} f^{p}(x) \mathrm{d} x
$$

where the constant $(p /(p-1))^{p}$ is best possible. 
Hardy inequality (2) has been generalized by G. H. Hardy himself in [8]. There he showed that for any integral function $f(x)>0$ on $(0, \infty), p>1$,

$$
\int_{0}^{\infty} \frac{1}{x^{m}}\left(\int_{x}^{\infty} f(t) \mathrm{d} t\right)^{p} \mathrm{~d} x \leq\left(\frac{p}{1-m}\right)^{p} \int_{0}^{\infty} \frac{1}{x^{m-p}} f^{p}(x) \mathrm{d} x, \quad m<1,
$$

$$
\int_{0}^{\infty} \frac{1}{x^{m}}\left(\int_{0}^{x} f(t) \mathrm{d} t\right)^{p} \mathrm{~d} x \leq\left(\frac{p}{m-1}\right)^{p} \int_{0}^{\infty} \frac{1}{x^{m-p}} f^{p}(x) \mathrm{d} x, \quad m>1 .
$$

Littlewood and Hardy [9] established the discrete version of (3) and (4).

Since the discovery of these two inequalities, various papers which deal with new proofs, generalizations, and extensions have appeared in the literature, $[6,7,10-12]$ are referred to readers. During the last decades, these inequalities were extended, a time scale version of weighted Hardy-type inequality is proved in [11], and some preliminary dynamic inequalities [13] are proved in time scales calculus. In $[14,15]$, some classical inequalities are proved for isotonic linear functionals and superquadratic functions.

Recently, many new Hardy inequalities have been proved, including Hardy and Rellich inequalities for Bessel pairs [16], Hardy-Sobolev inequalities on hypersurfaces for Euclidean space [17], improved Hardy inequalities with exact remainder terms [18], Hardy inequalities with double singular weights [19], and Hardy inequalities for class functions in one-dimensional fractional Orlicz-Sobolev spaces [20]. A new approach for the fractional integral operator in time scales with variable exponent Lebesgue spaces is presented in [21] and $n$-dimensional integral-type inequalities via time scale calculus is studied in [22].

\section{Preliminaries}

2.1. Some Basics on Time Scales. A time scale is a closed subset of the real line $\mathbb{R}$, and its common notation is $\mathbb{T}$. A time scale $\mathbb{T}$ may or may not be connected. Forward jump operator and backward jump operator are respectively defined as

$$
\begin{aligned}
& \sigma(\omega)=\inf \{s \in \mathbb{T}: s>\omega\}, \\
& \rho(\omega)=\sup \{s \in \mathbb{T}: s<\omega\} .
\end{aligned}
$$

In general, $\sigma(\omega) \geq \omega$ and $\rho(\omega) \leq \omega$. The forward and backward graininess functions $\mu, v: \mathbb{T} \longrightarrow[0,+\infty)$ are, respectively, defined by

$$
\begin{aligned}
& \mu(\omega)=\sigma(\omega)-\omega, \\
& \nu(\omega)=\omega-\rho(\omega) .
\end{aligned}
$$

If $\sigma(\omega)>\omega$, then $\omega$ is said to be right scattered. If $\rho(\omega)<\omega$, then $\omega$ is said to be left scattered. Points that are left-scattered and right scattered at the same time are called isolated. If $\omega<\sup \mathbb{T}$ and $\sigma(\omega)=\omega$, then $\omega$ is called the right-dense and if $\omega>\inf \mathbb{T}$ and $\rho(\omega)=\omega$, then $\omega$ is called left-dense. Points that are left-dense and right-dense at the same time are called dense.

Rd-continuous function: a function $\hbar: \mathbb{T} \longrightarrow \mathbb{R}$ is called rd-continuous if it is continuous at right-dense points in $\mathbb{T}$ and its left-sided limits exist at left-dense points in $\mathbb{T}$.

Notation: if $\mathbb{T}$ is a time scale, then the set $\mathbb{T}^{k}$ is defined as

$$
\mathbb{T}^{k}= \begin{cases}\frac{\mathbb{T}}{(\rho \sup \mathbb{T}, \sup \mathbb{}]}, & \sup \mathbb{}<\infty ; \\ \mathbb{T}, & \sup \mathbb{\mathbb { N }}=\infty .\end{cases}
$$

Delta derivatives: take a function $\hbar: \mathbb{T} \longrightarrow \mathbb{R}$. The delta derivative (Hilger derivative) $\hbar^{\Delta}(\xi)$ exists iff, for every $\mathfrak{\Im}>0$, there exists a neighborhood $\aleph$ of $\xi$ such that

$$
\left|\hbar(\sigma(\xi))-\hbar(s)-\hbar^{\Delta}(\xi)(\sigma(\xi)-s)\right| \leq \mathfrak{J}|\sigma(\xi)-s|,
$$

for all $s \in \aleph, \xi \in \mathbb{T}$.

\subsection{Some Principles of Fractional Calculus}

2.2.1. Riemann-Liouville Derivative. Riemann-Liouville derivative is the generalization of the usual derivative. It is based on Cauchy's formula for calculating iterated integrals, if the first integral of a function, which must be equal to deriving it to -1 , is as follows:

$$
D^{-1} \hbar(\vartheta)=\int_{0}^{\vartheta} \hbar(\omega) \mathrm{d} \omega \text {. }
$$

The calculation of the second can be simplified by interchanging the integration order,

$$
\begin{aligned}
D^{-2} \hbar(\vartheta) & =\int_{0}^{\vartheta} \int_{0}^{\omega_{2}} \hbar\left(\omega_{1}\right) \mathrm{d} \omega_{1} \mathrm{~d} \omega_{2} \\
& =\int_{0}^{\vartheta} \int_{\omega_{1}}^{\vartheta} \hbar\left(\omega_{1}\right) \mathrm{d} \omega_{2} \mathrm{~d} \omega_{1} \\
& =\int_{0}^{\vartheta} \hbar\left(\omega_{1}\right) \int_{\omega_{1}}^{\vartheta} \mathrm{d} \omega_{2} \mathrm{~d} \omega_{1} \\
& =\int_{0}^{\vartheta} \hbar(\omega)(\vartheta-\omega) \mathrm{d} \omega .
\end{aligned}
$$

This method can be applied repeatedly, resulting in the following formula for calculating iterated integrals,

$$
D^{-n} \hbar(\vartheta)=\frac{1}{(n-1) !} \int_{0}^{\vartheta} \hbar(\omega)(\vartheta-\omega)^{n-1} \mathrm{~d} \omega .
$$

Now, this can be easily generalized to noninteger values, in what is the Riemann-Liouville derivative [23],

$$
D^{\gamma} \hbar(\vartheta)=\frac{1}{\Gamma(-\gamma)} \int_{0}^{\vartheta} \frac{\hbar(\omega)}{(\vartheta-\omega)^{\gamma+1}} \mathrm{~d} \omega \text {. }
$$

The Riemann-Liouville derivative with the lower integration limit $a$ would be [23] 


$$
{ }_{a} D_{\vartheta}^{\gamma} \hbar(\vartheta)=\frac{1}{\Gamma(-\gamma)} \int_{a}^{\vartheta} \frac{\hbar(\omega)}{(\vartheta-\omega)^{\gamma+1}} \mathrm{~d} \omega .
$$

2.2.2. Riemann-Liouville Integral. The Riemann-Liouville integral relates with a real function $\hbar: \mathbb{R} \longrightarrow \mathbb{R}$, another same kind of function $I^{\gamma} \hbar$ for each value of the parameter $\gamma>0$. The integral is a generalization of the repeated antiderivative of $\hbar$ of order real number $\gamma$.

Let $J=[a, b](-\infty<a<b<\infty)$ be a finite interval of $\mathbb{R}$. The left and right Riemann-Liouville fractional integrals ${ }_{a} D_{\omega}^{-\gamma} \hbar$ and ${ }_{t} D_{b}^{-\gamma} \hbar$ of order $\gamma \in \mathbb{R}^{+}$are defined in [23] by

$$
\begin{aligned}
& { }_{a} D_{\omega}^{-\gamma} \hbar(\omega)=\frac{1}{\Gamma(\gamma)} \int_{a}^{\omega}(\omega-s)^{\gamma-1} \hbar(s) \mathrm{d} s, \quad \omega>a, \gamma>0, \\
& { }_{t} D_{b}^{-\gamma} \hbar(\omega)=\frac{1}{\Gamma(\gamma)} \int_{\omega}^{b}(s-\omega)^{\gamma-1} \hbar(s) \mathrm{d} s, \quad \omega<b, \gamma>0,
\end{aligned}
$$

respectively, provided the right-hand sides are pointwise defined on $[a, b]$. When $\gamma=n \in N^{+}$, definitions (14) and (15) coincide with the nth integrals of the form,

$$
\begin{aligned}
& { }_{a} D_{\omega}^{-n} \hbar(\omega)=\frac{1}{(n-1) !} \int_{a}^{\omega}(\omega-s)^{n-1} \hbar(s) \mathrm{d} s, \\
& { }_{t} D_{b}^{-n} \hbar(\omega)=\frac{1}{(n-1) !} \int_{\omega}^{b}(s-\omega)^{n-1} \hbar(s) \mathrm{d} s .
\end{aligned}
$$

2.3. Some Principles of Delta Fractional Calculus. For $\mu, \nu>0$, one has that (see [24], p. 256)

$$
\int_{u}^{\xi}\left(\frac{(\xi-r)^{\ell-1}}{\Gamma(\ell)}\right)\left(\frac{(r-u)^{\mathscr{g}-1}}{\Gamma(\mathcal{J})}\right) \mathrm{d} r=\frac{(\xi-u)^{\ell+\mathscr{J}-1}}{\Gamma(\ell+\mathcal{J})},
$$

where $\Gamma$ is the Gamma function. If time scale $\mathbb{T}$ is considered to be $\mathbb{T}=\mathbb{T}^{k}$, then the coordinate-wise rd-continuous functions are given by

$$
h_{\gamma}: \mathbb{T} \times \mathbb{T} \longrightarrow \mathbb{R}, \quad \gamma \geq 0,
$$

such that $h_{0}(\xi, r)=1, \forall \xi, r \in \mathbb{T}$ and

$$
h_{\gamma+1}(\xi, r)=\int_{r}^{\xi} h_{\gamma}(y, r) \Delta y, \quad \forall \xi, r, y \in \mathbb{T} .
$$

Furthermore, for $\gamma, \beta>1$, it is assumed that

$\int_{\sigma(r)}^{\xi} h_{\gamma-1}(\xi, \sigma(s)) h_{\beta-1}(s, \sigma(r)) \Delta s=h_{\gamma+\beta-1}(\xi, \sigma(r)), \quad \forall r<\xi ; r, \xi \in \mathbb{T}$.

In the case $\mathbb{T}=\mathbb{R}$ and for $\sigma(r)=r$, one obtains

$$
\begin{aligned}
& h_{k}(r, \xi)=\frac{(r-\xi)^{\kappa}}{\kappa !}, \quad \kappa \in \mathbb{N}_{0}=\mathbb{N} \cup\{0\}, \\
& h_{\gamma}(r, \xi)=\frac{(r-\xi)^{\gamma}}{\Gamma(\gamma+1)}, \quad \gamma \geq 0,
\end{aligned}
$$

and also

$$
\int_{r}^{\xi} \frac{(\xi-r)^{\gamma}}{\Gamma(\gamma+1)} \mathrm{d} \xi=\frac{(\xi-r)^{\gamma+1}}{\Gamma(\gamma+2)}=h_{\gamma+1}(\xi, r),
$$

satisfying (19). Furthermore, it is observed that for $\gamma, \beta>1$ and by using (17), one gets

$$
\begin{aligned}
& \int_{r}^{\varsigma} h_{\gamma-1}(\varsigma, \xi) h_{\beta-1}(\xi, \Phi) \mathrm{d} \xi \\
& =\int_{r}^{\varsigma} \frac{(\varsigma-\xi)^{\gamma-1}}{\Gamma(\gamma)} \frac{(\xi-\Phi)^{\beta-1}}{\Gamma(\beta)} \mathrm{d} \xi \\
& =\frac{(\varsigma-\Phi)^{\gamma+\beta-1}}{\Gamma(\gamma+\beta)} \\
& =h_{\gamma+\beta-1}(\varsigma, \Phi),
\end{aligned}
$$

which is satisfying (20).

If $g \in C_{\mathrm{rd}}$ and $x \in \mathbb{T}^{k}$ ([25], Chap: 1$)$, then

$$
\int_{x}^{\sigma(x)} g(\varsigma) \Delta \varsigma=\mu(x) g(x) \text {. }
$$

2.3.1. Riemann-Liouville Delta Fractional Integral. For $\gamma \geq 1$, the $\Delta$-Riemann-Liouville type fractional integral is defined in [26] as

$$
K_{a}^{\gamma} \phi(\omega)=\int_{a}^{\omega} h_{\gamma-1}(\omega, \sigma(\nu)) \phi(\nu) \Delta \nu,
$$

for $[a, b] \in \mathbb{T}$ and $\omega \in[a, b] \cap \mathbb{T}$. Notice that $K_{a}^{0} \phi=\phi$, where $\phi \in L_{1}([a, b] \cap \mathbb{T})$ (Lebesgue $\Delta$-integrable functions on $[a, b] \cap \mathbb{T},[27])$.

2.3.2. Fubini's Theorem on Time Scale. Consider $r, l \in \mathbb{T}$ and $\phi \in C_{r d}(\mathbb{T} \times \mathbb{T}, \mathbb{R})$. Then,

$$
\int_{r}^{l} \int_{\eta}^{l} \phi(\eta, \zeta) \Delta \zeta \Delta \eta=\int_{r}^{l} \int_{r}^{\sigma(\zeta)} \phi(\eta, \zeta) \Delta \eta \Delta \zeta .
$$

It can be found in [28].

\subsection{Superquadratic Function}

Definition 1. A function $\Theta:[0, \infty) \longrightarrow \mathbb{R}$ is called superquadratic 29 provided that, for all $\omega \geq 0$, there exists a constant $C(\omega) \in \mathbb{R}$ such that

$$
\Theta(\nu)-\Theta(\omega)-\Theta(|\nu-\omega|) \geq C(\omega)(\nu-\omega),
$$


for all $\nu \geq 0$. We say that $\phi$ is subquadratic if $-\phi$ is a superquadratic function.

The following lemma (see [29]) shows that every positive superquadratic function is also a convex function.

Lemma 1. Let $\Theta$ be a superquadratic function with $C(\omega)$ as in Definition 1. Then,

(i) $\Theta(0) \leq 0$.

(ii) If $\Theta(0)=\Theta^{\prime}(0)=0$, then $C(\omega)=\Theta^{\prime}(\omega)$, where $\Theta$ is differentiable at $\omega>0$.

(iii) If $\Theta \geq 0$, then $\Theta$ is convex and $\Theta(0)=\Theta^{\prime}(0)=0$.

\section{Jensen Inequality for Superquadratic Functions via Delta Fractional Integrals}

Theorem 1. Let $h_{\gamma-1}: \mathbb{T} \times \mathbb{T} \longrightarrow \mathbb{R}$ where $\gamma \geq 1$ and let $\vartheta_{1} \in \mathbb{T}$. If $\Upsilon_{1}=\left[\lambda_{1}, \lambda_{2}\right] \cap \mathbb{T}$ with $\lambda_{1}, \lambda_{2} \in T$ and $\phi: \Upsilon_{1} \longrightarrow[0, \infty)$ is rd-continuous, then for every continuous superquadratic function $\Theta:[0, \infty) \longrightarrow \mathbb{R}$,

$\Theta\left(\frac{K_{\lambda_{1}}^{\gamma} \phi\left(\vartheta_{1}\right)}{\mathrm{F}\left(\vartheta_{1}\right)}\right) \leq \frac{1}{\mathrm{~F}\left(\vartheta_{1}\right)} \int_{\lambda_{1}}^{\vartheta_{1}}\left(\Theta(\phi(s))-\Theta\left(\left|\phi(s)-\frac{K_{\lambda_{1}}^{\gamma} \phi\left(\vartheta_{1}\right)}{\mathrm{F}(s)}\right|\right)\right) \Delta s$,

where

$$
\mathrm{F}\left(\vartheta_{1}\right)=\int_{\lambda_{1}}^{\vartheta_{1}} h_{\gamma-1}\left(\vartheta_{1}, \sigma\left(\vartheta_{2}\right)\right) \Delta \vartheta_{2}
$$

Proof. Let $\Theta:[0, \infty) \longrightarrow \mathbb{R}$ be a superquadratic function and $\vartheta_{0} \in[0, \infty)$. According to (27), there is a constant $C\left(\vartheta_{0}\right)$ such that

$$
\Theta\left(\vartheta_{2}\right) \geq \Theta\left(\vartheta_{0}\right)+C\left(\vartheta_{0}\right)\left(\vartheta_{2}-\vartheta_{0}\right)+\Theta\left(\left|\vartheta_{2}-\vartheta_{0}\right|\right) .
$$

Since $\phi$ is rd-continuous,

$$
\vartheta_{0}=\frac{K_{\lambda_{1}}^{\gamma} \phi\left(\vartheta_{1}\right)}{F\left(\vartheta_{1}\right)}
$$

is well defined. The function $\Theta \circ \phi$ is also rd-continuous, so we may apply (30) with $\vartheta_{2}=\phi(s)$ and (31) to obtain

$$
\begin{aligned}
\Theta(\phi(s)) \geq & \Theta\left(\frac{K_{\lambda_{1}}^{\gamma} \phi\left(\vartheta_{1}\right)}{F\left(\vartheta_{1}\right)}\right)+C\left(\vartheta_{0}\right)\left(\phi(s)-\frac{K_{\lambda_{1}}^{\gamma} \phi\left(\vartheta_{1}\right)}{F\left(\vartheta_{1}\right)}\right) \\
& +\Theta\left(\left|\phi(s)-\frac{K_{\lambda_{1}}^{\gamma} \phi\left(\vartheta_{1}\right)}{F\left(\vartheta_{1}\right)}\right|\right) .
\end{aligned}
$$

Integrating from $\lambda_{1}$ to $\vartheta_{1}$, we get

$$
\begin{aligned}
& \int_{\lambda_{1}}^{\vartheta_{1}} h_{\gamma-1}\left(\vartheta_{1}, \sigma(s)\right) \Theta(\phi(s)) \Delta s-\int_{\lambda_{1}}^{\vartheta_{1}} h_{\gamma-1}\left(\vartheta_{1}, \sigma(s)\right) \Theta\left(\left|\phi(s)-\frac{K_{\lambda_{1}}^{\gamma} \phi\left(\vartheta_{1}\right)}{F\left(\vartheta_{1}\right)}\right|\right) \Delta s \\
& \quad-\int_{\lambda_{1}}^{\vartheta_{1}} h_{\gamma-1}\left(\vartheta_{1}, \sigma(s)\right) \Theta\left(\frac{K_{\lambda_{1}}^{\gamma} \phi\left(\vartheta_{1}\right)}{F\left(\vartheta_{1}\right)}\right) \Delta s \\
& \geq C\left(\vartheta_{0}\right) \int_{\lambda_{1}}^{\vartheta_{1}} h_{\gamma-1}\left(\vartheta_{1}, \sigma(s)\right)\left(\phi(s)-\frac{K_{\lambda_{1}}^{\gamma} \phi\left(\vartheta_{1}\right)}{F\left(\vartheta_{1}\right)}\right) \Delta s \\
& =C\left(\vartheta_{0}\right)\left(K_{\lambda_{1}}^{\gamma} \phi\left(\vartheta_{1}\right)-\int_{\lambda_{1}}^{\vartheta_{1}} h_{\gamma-1}\left(\vartheta_{1}, \sigma(s)\right) \frac{K_{\lambda_{1}}^{\gamma} \phi\left(\vartheta_{1}\right)}{F\left(\vartheta_{1}\right)} \Delta s\right) \\
& =C\left(\vartheta_{0}\right)\left(K_{\lambda_{1}}^{\gamma} \phi\left(\vartheta_{1}\right)-F\left(\vartheta_{1}\right) \cdot \frac{K_{\lambda_{1}}^{\gamma} \phi\left(\vartheta_{1}\right)}{F\left(\vartheta_{1}\right)}\right) \\
& =C\left(\vartheta_{0}\right)\left(K_{\lambda_{1}}^{\gamma} \phi\left(\vartheta_{1}\right)-K_{\lambda_{1}}^{\gamma} \phi\left(\vartheta_{1}\right)\right)=0 .
\end{aligned}
$$

Remark 1. Since $\Theta$ is nonnegative superquadratic function, therefore (by Lemma 1) a convex one too, the result of (28) refines the result given in [30] (Theorem 1) and by assuming $\gamma=1$, (28) becomes [31] (Theorem 2.5), and result of [31] (Theorem 2.5) refines the result given in [31] (Theorem 2.4). Furthermore, respective inequality is obtained in fractional calculus ([29], Theorem 2.3) by assuming $\gamma=1$ and $\mathbb{T}=\mathbb{R}$. 
Example 1. If $\mathbb{T}=\mathbb{N}$, then $\sigma\left(\vartheta_{2}\right)=\vartheta_{2}+1, \forall \vartheta_{2} \in \mathbb{N}$, and By substituting (28) and (34), it becomes

$$
h_{\gamma-1}\left(\vartheta_{1}, \sigma\left(\vartheta_{2}\right)\right)=\frac{\left(\vartheta_{1}-\sigma\left(\vartheta_{2}\right)\right)^{\gamma-1}}{(\gamma-1) !}=\frac{\left(\vartheta_{1}-\vartheta_{2}-1\right)^{\gamma-1}}{(\gamma-1) !} \text {. }
$$

$$
\Theta\left(\frac{\sum_{\vartheta_{2}=\lambda_{1}}^{\vartheta_{1}-1}\left(\vartheta_{1}-\vartheta_{2}-1\right)^{\gamma-1} \phi\left(\vartheta_{2}\right)}{\sum_{\vartheta_{2}=\lambda_{1}}^{\vartheta_{1}-1}\left(\vartheta_{1}-\vartheta_{2}-1\right)^{\gamma-1}}\right) \leq \frac{(\gamma-1) !}{\sum_{\vartheta_{2}=\lambda_{1}}^{\vartheta_{1}-1}\left(\vartheta_{1}-\vartheta_{2}-1\right)^{\gamma-1}} \sum_{s=\lambda_{1}}^{\vartheta_{1}-1}\left(\Theta(\phi(s))-\Theta\left(\left|\phi(s)-\frac{\sum_{\vartheta_{2}=\lambda_{1}}^{\vartheta_{1}-1}\left(\vartheta_{1}-\vartheta_{2}-1\right)^{\gamma-1} \phi\left(\vartheta_{2}\right)}{\sum_{\vartheta_{2}=\lambda_{1}}^{\vartheta_{1}-1}\left(\vartheta_{1}-\vartheta_{2}-1\right)^{\gamma-1}}\right|\right)\right)
$$

Example 2. If $\mathbb{T}=q^{\mathbb{Z}}$, then $\sigma\left(\vartheta_{2}\right)=q \vartheta_{2}$. In this case,

$$
h_{\gamma-1}\left(\vartheta_{1}, \sigma\left(\vartheta_{2}\right)\right)=\prod_{v=0}^{\gamma-2} \frac{\vartheta_{1}-q^{v} \sigma\left(\vartheta_{2}\right)}{\sum_{\mu=0}^{v} q^{\mu}}=\prod_{v=0}^{\gamma-2} \frac{\vartheta_{1}-q^{v+1} \vartheta_{2}}{\sum_{\mu=0}^{v} q^{\mu}}
$$

$$
\begin{aligned}
& \Theta\left(\frac{\sum_{\vartheta_{2}=\lambda_{1}}^{\vartheta_{1}-1}\left(\prod_{v=0}^{\gamma-2}\left(\vartheta_{1}-q^{v+1} \vartheta_{2} / \sum_{\mu=0}^{v} q^{\mu}\right)\right) \phi\left(\vartheta_{2}\right)}{\sum_{\vartheta_{2}=\lambda_{1}}^{\vartheta_{1}-1}\left(\prod_{v=0}^{\gamma-2}\left(\vartheta_{1}-q^{v+1} \vartheta_{2} / \sum_{\mu=0}^{v} q^{\mu}\right)\right)}\right) \\
& \leq \frac{1}{\prod_{v=0}^{\gamma-2}\left(\vartheta_{1}-q^{v+1} \vartheta_{2} / \sum_{\mu=0}^{v} q^{\mu}\right)} \sum_{s=\lambda_{1}}^{\vartheta_{1}-1}\left(\Theta(\phi(s))-\Theta\left(\left|\phi(s)-\frac{\sum_{\vartheta_{2}=\lambda_{1}}^{\vartheta_{1}-1}\left(\prod_{v=0}^{\gamma-2}\left(\vartheta_{1}-q^{v+1} \vartheta_{2} / \sum_{\mu=0}^{v} q^{\mu}\right)\right) \phi\left(\vartheta_{2}\right)}{\sum_{\vartheta_{2}=\lambda_{1}}^{\vartheta_{1}-1}\left(\prod_{v=0}^{\gamma-2}\left(\vartheta_{1}-q^{v+1} \vartheta_{2} / \sum_{\mu=0}^{v} q^{\mu}\right)\right)}\right|\right)\right)
\end{aligned}
$$

\section{Hardy Inequalities for Superquadratic Functions with General Kernels} and $\eta_{1}<s_{2}<\eta_{2}$. Define

Theorem 2. Assume $h_{\gamma-1}: \mathbb{T} \times \mathbb{T} \longrightarrow \mathbb{R}$ where $\gamma \geq 1, \beta \geq 1$, and $\Upsilon_{1}=\left[\lambda_{1}, \lambda_{2}\right] \cap \mathbb{T}$ and $\Upsilon_{2}=\left[\eta_{1}, \eta_{2}\right] \cap T$ where

$$
A_{\kappa}\left(\phi\left(\vartheta_{1}\right)\right)=\frac{1}{\chi\left(\vartheta_{1}\right)}\left(\int_{\lambda_{1}}^{s_{1}} h_{\gamma-1}\left(s_{1}, \sigma\left(\vartheta_{2}\right)\right) \kappa\left(\vartheta_{1}, \vartheta_{2}\right) \phi\left(\vartheta_{2}\right) \Delta \vartheta_{2}-\int_{\lambda_{2}}^{s_{1}} h_{\gamma-1}\left(s_{1}, \sigma\left(\vartheta_{2}\right)\right) \kappa\left(\vartheta_{1}, \vartheta_{2}\right) \phi\left(\vartheta_{2}\right) \Delta \vartheta_{2}\right),
$$

where $\phi: \Upsilon_{1} \longrightarrow \mathbb{R}$ is a $\lambda_{\Delta}$-measurable function, $\kappa: \Upsilon_{2} \times \Upsilon_{1} \longrightarrow \mathbb{R}$ is a nonnegative kernel, and

$$
\chi\left(\vartheta_{1}\right)=\left(\int_{\lambda_{1}}^{\vartheta_{1}} h_{\gamma-1}\left(\vartheta_{1}, \sigma\left(\vartheta_{2}\right)\right) \kappa\left(\vartheta_{1}, \vartheta_{2}\right) \Delta \vartheta_{2}-\int_{\lambda_{2}}^{\vartheta_{1}} h_{\gamma-1}\left(\vartheta_{1}, \sigma\left(\vartheta_{2}\right)\right) \kappa\left(\vartheta_{1}, \vartheta_{2}\right) \Delta \vartheta_{2}\right)<\infty, \quad \vartheta_{1} \in \Upsilon_{2} .
$$

Let $\zeta: \Upsilon_{2} \longrightarrow \mathbb{R}_{+}$be $\mu_{\Delta}$-integrable function. Denote

$$
w\left(\vartheta_{2}\right)=\int_{\eta_{1}}^{\eta_{2}} \frac{\kappa\left(\vartheta_{1}, \vartheta_{2}\right) \zeta\left(\vartheta_{1}\right)}{\chi\left(\vartheta_{1}\right)} \Delta \vartheta_{1}, \quad \vartheta_{2} \in \Upsilon_{1}
$$

Further assume $I=[a, c), 0 \leq a<c \leq \infty$, and $\Theta: I \longrightarrow R$. If $\Theta \in C(I, R)$ is superquadratic, then 


$$
\begin{aligned}
& \int_{\eta_{1}}^{\eta_{2}} h_{\beta-1}\left(s_{2}, \sigma\left(\vartheta_{1}\right)\right) \Theta\left(A_{\kappa}\left(\phi\left(\vartheta_{1}\right)\right)\right) \zeta\left(\vartheta_{1}\right) \Delta \vartheta_{1} \\
& \quad+\int_{\lambda_{1}}^{\lambda_{2}} h_{\gamma-1}\left(s_{1}, \sigma\left(\vartheta_{2}\right)\right) h_{\beta-1}\left(s_{2}, \sigma\left(\vartheta_{1}\right)\right)\left(\int_{\eta_{1}}^{\eta_{2}} \Theta\left(\left|\phi\left(\vartheta_{2}\right)-A_{\kappa}\left(\phi\left(\vartheta_{1}\right)\right)\right|\right) \frac{\zeta\left(\vartheta_{1}\right) \kappa\left(\vartheta_{1}, \vartheta_{2}\right)}{\chi\left(\vartheta_{1}\right)} \Delta \vartheta_{1}\right) \Delta \vartheta_{2} \\
& \leq \int_{\lambda_{1}}^{\lambda_{2}} h_{\gamma-1}\left(s_{1}, \sigma\left(\vartheta_{2}\right)\right) h_{\beta-1}\left(s_{2}, \sigma\left(\vartheta_{1}\right)\right) \Theta\left(\phi\left(\vartheta_{1}\right)\right) w\left(\vartheta_{2}\right) \Delta \vartheta_{2},
\end{aligned}
$$

holds for all $\lambda_{\Delta}$-integrable $\phi: \Upsilon_{1} \longrightarrow \mathbb{R}$ such that $\phi\left(\Upsilon_{1}\right) \subset I$. Proof. Consider If $\Theta$ is subquadratic, then inequality (41) is reversed.

$$
\begin{aligned}
& \int_{\eta_{1}}^{\eta_{2}} h_{\beta-1}\left(s_{2}, \sigma\left(\vartheta_{1}\right)\right) \Theta\left(A_{\kappa}\left(\phi\left(\vartheta_{1}\right)\right)\right) \zeta\left(\vartheta_{1}\right) \Delta \vartheta_{1} \\
& =\int_{\eta_{1}}^{\eta_{2}} h_{\beta-1}\left(s_{2}, \sigma\left(\vartheta_{1}\right)\right) \zeta\left(\vartheta_{1}\right) \times \Theta\left(\frac{1}{\chi\left(\vartheta_{1}\right)}\left(\int_{\lambda_{1}}^{s_{1}} h_{\gamma-1}\left(s_{1}, \sigma\left(\vartheta_{2}\right)\right) \kappa\left(\vartheta_{1}, \vartheta_{2}\right) \phi\left(\vartheta_{2}\right) \Delta \vartheta_{2}-\int_{\lambda_{2}}^{s_{1}} h_{\gamma-1}\left(s_{1}, \sigma\left(\vartheta_{2}\right)\right) \kappa\left(\vartheta_{1}, \vartheta_{2}\right) \phi\left(\vartheta_{2}\right) \Delta \vartheta_{2}\right)\right) \Delta \vartheta_{1} \\
& =\int_{\eta_{1}}^{\eta_{2}} h_{\beta-1}\left(s_{2}, \sigma\left(\vartheta_{1}\right)\right) \zeta\left(\vartheta_{1}\right) \Theta\left(\frac{1}{\chi\left(\vartheta_{1}\right)} \int_{\lambda_{1}}^{s_{1}} h_{\gamma-1}\left(s_{1}, \sigma\left(\vartheta_{2}\right)\right) \kappa\left(\vartheta_{1}, \vartheta_{2}\right) \phi\left(\vartheta_{2}\right) \Delta \vartheta_{2}\right) \Delta \vartheta_{1} \\
& \quad-\int_{\eta_{1}}^{\eta_{2}} h_{\beta-1}\left(s_{2}, \sigma\left(\vartheta_{1}\right)\right) \zeta\left(\vartheta_{1}\right) \Theta\left(\frac{1}{\chi\left(\vartheta_{1}\right)} \int_{\lambda_{2}}^{s_{1}} h_{\gamma-1}\left(s_{1}, \sigma\left(\vartheta_{2}\right)\right) \kappa\left(\vartheta_{1}, \vartheta_{2}\right) \phi\left(\vartheta_{2}\right) \Delta \vartheta_{2}\right) \Delta \vartheta_{1} .
\end{aligned}
$$

By using Jensen's inequality (28),

$$
\begin{aligned}
& \leq \int_{\eta_{1}}^{\eta_{2}} h_{\beta-1}\left(s_{2}, \sigma\left(\vartheta_{1}\right)\right) \frac{\zeta\left(\vartheta_{1}\right)}{\chi\left(\vartheta_{1}\right)}\left(\int_{\lambda_{1}}^{s_{1}} h_{\gamma-1}\left(s_{1}, \sigma\left(\vartheta_{2}\right)\right) \kappa\left(\vartheta_{1}, \vartheta_{2}\right) \Theta\left(\phi\left(\vartheta_{2}\right)\right) \Delta \vartheta_{2}\right) \Delta \vartheta_{1} \\
& -\int_{\eta_{1}}^{\eta_{2}} h_{\beta-1}\left(s_{2}, \sigma\left(\vartheta_{1}\right)\right) \frac{\zeta\left(\vartheta_{1}\right)}{\chi\left(\vartheta_{1}\right)}\left(\int_{\lambda_{1}}^{s_{1}} h_{\gamma-1}\left(s_{1}, \sigma\left(\vartheta_{2}\right)\right) \kappa\left(\vartheta_{1}, \vartheta_{2}\right) \Theta\left(\left|\phi\left(\vartheta_{2}\right)-A_{\kappa}\left(\phi\left(\vartheta_{1}\right)\right)\right|\right) \Delta \vartheta_{2}\right) \Delta \vartheta_{1} \\
& -\int_{\eta_{1}}^{\eta_{2}} h_{\beta-1}\left(s_{2}, \sigma\left(\vartheta_{1}\right)\right) \frac{\zeta\left(\vartheta_{1}\right)}{\chi\left(\vartheta_{1}\right)}\left(\int_{\lambda_{2}}^{s_{1}} h_{\gamma-1}\left(s_{1}, \sigma\left(\vartheta_{2}\right)\right) \kappa\left(\vartheta_{1}, \vartheta_{2}\right) \Theta\left(\phi\left(\vartheta_{2}\right)\right) \Delta \vartheta_{2}\right) \Delta \vartheta_{1} \\
& +\int_{\eta_{1}}^{\eta_{2}} h_{\beta-1}\left(s_{2}, \sigma\left(\vartheta_{1}\right)\right) \frac{\zeta\left(\vartheta_{1}\right)}{\chi\left(\vartheta_{1}\right)}\left(\int_{\lambda_{2}}^{s_{1}} h_{\gamma-1}\left(s_{1}, \sigma\left(\vartheta_{2}\right)\right) \kappa\left(\vartheta_{1}, \vartheta_{2}\right) \Theta\left(\left|\phi\left(\vartheta_{2}\right)-A_{\kappa}\left(\phi\left(\vartheta_{1}\right)\right)\right|\right) \Delta \vartheta_{2}\right) \Delta \vartheta_{1} \\
& \leq \int_{\eta_{1}}^{\eta_{2}} h_{\beta-1}\left(s_{2}, \sigma\left(\vartheta_{1}\right)\right) \frac{\zeta\left(\vartheta_{1}\right)}{\chi\left(\vartheta_{1}\right)}\left(\int_{\lambda_{1}}^{s_{1}} h_{\gamma-1}\left(s_{1}, \sigma\left(\vartheta_{2}\right)\right) \kappa\left(\vartheta_{1}, \vartheta_{2}\right) \Theta\left(\phi\left(\vartheta_{2}\right)\right) \Delta \vartheta_{2}-\int_{\lambda_{2}}^{s_{1}} h_{\gamma-1}\left(s_{1}, \sigma\left(\vartheta_{2}\right)\right) \kappa\left(\vartheta_{1}, \vartheta_{2}\right) \Theta\left(\phi\left(\vartheta_{2}\right)\right) \Delta \vartheta_{2}\right) \Delta \vartheta_{1} \\
& -\int_{\eta_{1}}^{\eta_{2}} h_{\beta-1}\left(s_{2}, \sigma\left(\vartheta_{1}\right)\right) \frac{\zeta\left(\vartheta_{1}\right)}{\chi\left(\vartheta_{1}\right)}\left(\int_{\lambda_{1}-\int_{\lambda_{2}}^{s_{1}} h_{\gamma-1}^{s_{1}}\left(s_{1}, \sigma\left(\vartheta_{2}\right)\right) \kappa\left(\vartheta_{1}, \vartheta_{2}\right) \Theta\left(\left|\phi\left(\vartheta_{2}\right)-A_{\kappa}\left(\phi\left(\vartheta_{1}\right)\right)\right|\right) \Delta \vartheta_{2} \Delta \vartheta_{1}}^{h_{1-1}\left(s_{1}, \sigma\left(\vartheta_{2}\right)\right) \kappa\left(\vartheta_{1}, \vartheta_{2}\right) \Theta\left(\left|\phi\left(\vartheta_{2}\right)-A_{\kappa}\left(\phi\left(\vartheta_{1}\right)\right)\right|\right) \Delta \vartheta_{2}}\right) \\
& \leq \int_{\eta_{1}}^{\eta_{2}} h_{\beta-1}\left(s_{2}, \sigma\left(\vartheta_{1}\right)\right) \frac{\zeta\left(\vartheta_{1}\right)}{\chi\left(\vartheta_{1}\right)}\left(\int_{\lambda_{1}}^{\lambda_{2}} h_{\gamma-1}\left(s_{1}, \sigma\left(\vartheta_{2}\right)\right) \kappa\left(\vartheta_{1}, \vartheta_{2}\right) \Theta\left(\phi\left(\vartheta_{2}\right)\right) \Delta \vartheta_{2}\right) \Delta \vartheta_{1} \\
& -\int_{\eta_{1}}^{\eta_{2}} h_{\beta-1}\left(s_{2}, \sigma\left(\vartheta_{1}\right)\right) \frac{\zeta\left(\vartheta_{1}\right)}{\chi\left(\vartheta_{1}\right)}\left(\int_{\lambda_{1}}^{\lambda_{2}} h_{\gamma-1}\left(s_{1}, \sigma\left(\vartheta_{2}\right)\right) \kappa\left(\vartheta_{1}, \vartheta_{2}\right) \Theta\left(\left|\phi\left(\vartheta_{2}\right)-A_{\kappa}\left(\phi\left(\vartheta_{1}\right)\right)\right|\right) \Delta \vartheta_{2}\right) \Delta \vartheta_{1} .
\end{aligned}
$$


By using Fubini's theorem,

$$
\begin{aligned}
= & \int_{\lambda_{1}}^{s_{1}} h_{\gamma-1}\left(s_{1}, \sigma\left(\vartheta_{2}\right)\right) h_{\beta-1}\left(s_{2}, \sigma\left(\vartheta_{1}\right)\right) \Theta\left(\phi\left(\vartheta_{2}\right)\right)\left(\int_{\eta_{1}}^{\eta_{2}} \frac{\kappa\left(\vartheta_{1}, \vartheta_{2}\right) \zeta\left(\vartheta_{1}\right)}{\chi\left(\vartheta_{1}\right)} \Delta \vartheta_{1}\right) \Delta \vartheta_{2} \\
& -\int_{\lambda_{1}}^{s_{1}} h_{\gamma-1}\left(s_{1}, \sigma\left(\vartheta_{2}\right)\right) h_{\beta-1}\left(s_{2}, \sigma\left(\vartheta_{1}\right)\right)\left(\int_{\eta_{1}}^{\eta_{2}} \Theta\left(\left|\phi\left(\vartheta_{2}\right)-A_{\kappa}\left(\phi\left(\vartheta_{1}\right)\right)\right|\right) \frac{\kappa\left(\vartheta_{1}, \vartheta_{2}\right) \zeta\left(\vartheta_{1}\right)}{\chi\left(\vartheta_{1}\right)} \Delta \vartheta_{1}\right) \Delta \vartheta_{2} \\
& -\int_{\lambda_{1}}^{s_{2}} h_{\gamma-1}\left(s_{1}, \sigma\left(\vartheta_{2}\right)\right) h_{\beta-1}\left(s_{2}, \sigma\left(\vartheta_{1}\right)\right) \Theta\left(\phi\left(\vartheta_{2}\right)\right)\left(\int_{\eta_{1}}^{\eta_{2}} \frac{\kappa\left(\vartheta_{1}, \vartheta_{2}\right) \zeta\left(\vartheta_{1}\right)}{\chi\left(\vartheta_{1}\right)} \Delta \vartheta_{1}\right) \Delta \vartheta_{2} \\
& +\int_{\lambda_{1}}^{s_{2}} h_{\gamma-1}\left(s_{1}, \sigma\left(\vartheta_{2}\right)\right) h_{\beta-1}\left(s_{2}, \sigma\left(\vartheta_{1}\right)\right)\left(\int_{\eta_{1}}^{\eta_{2}} \Theta\left(\left|\phi\left(\vartheta_{2}\right)-A_{\kappa}\left(\phi\left(\vartheta_{1}\right)\right)\right|\right) \frac{\kappa\left(\vartheta_{1}, \vartheta_{2}\right) \zeta\left(\vartheta_{1}\right)}{\chi\left(\vartheta_{1}\right)} \Delta \vartheta_{1}\right) \Delta \vartheta_{2} \\
= & \int_{\lambda_{1}}^{\lambda_{2}} h_{\gamma-1}\left(s_{1}, \sigma\left(\vartheta_{2}\right)\right) h_{\beta-1}\left(s_{2}, \sigma\left(\vartheta_{1}\right)\right) \Theta\left(\phi\left(\vartheta_{2}\right)\right)\left(\int_{\eta_{1}}^{\eta_{2}} \frac{\kappa\left(\vartheta_{1}, \vartheta_{2}\right) \zeta\left(\vartheta_{1}\right)}{\chi\left(\vartheta_{1}\right)} \Delta \vartheta_{1}\right) \Delta \vartheta_{2} \\
& -\int_{\lambda_{1}}^{\lambda_{2}} h_{\gamma-1}\left(s_{1}, \sigma\left(\vartheta_{2}\right)\right) h_{\beta-1}\left(s_{2}, \sigma\left(\vartheta_{1}\right)\right)\left(\int_{\eta_{1}}^{\eta_{2}} \Theta\left(\left|\phi\left(\vartheta_{2}\right)-A_{\kappa}\left(\phi\left(\vartheta_{1}\right)\right)\right|\right) \frac{\kappa\left(\vartheta_{1}, \vartheta_{2}\right) \zeta\left(\vartheta_{1}\right)}{\chi\left(\vartheta_{1}\right)} \Delta \vartheta_{1}\right) \Delta \vartheta_{2} \\
= & \int_{\lambda_{1}}^{\lambda_{2}} h_{\gamma-1}\left(s_{1}, \sigma\left(\vartheta_{2}\right)\right) h_{\beta-1}\left(s_{2}, \sigma\left(\vartheta_{1}\right)\right) \Theta\left(\phi\left(\vartheta_{2}\right)\right) w\left(\vartheta_{2}\right) \Delta \vartheta_{2} \\
& -\int_{\lambda_{1}}^{\lambda_{2}} h_{\gamma-1}\left(s_{1}, \sigma\left(\vartheta_{2}\right)\right) h_{\beta-1}\left(s_{2}, \sigma\left(\vartheta_{1}\right)\right)\left(\int_{\eta_{1}}^{\eta_{2}} \Theta\left(\left|\phi\left(\vartheta_{2}\right)-A_{\kappa}\left(\phi\left(\vartheta_{1}\right)\right)\right|\right) \frac{\kappa\left(\vartheta_{1}, \vartheta_{2}\right) \zeta\left(\vartheta_{1}\right)}{\chi\left(\vartheta_{1}\right)} \Delta \vartheta_{1}\right) \Delta \vartheta_{2} .
\end{aligned}
$$

Remark 2. If the function $\Theta$ is subquadratic, then Jensen's inequality for superquadratic functions on delta fractional integrals is reversed, which implies, according to the conclusions made above, that inequality sign in (41) is reversed.

In [30], authors prove the analogous results for convex functions; now, it is easy to see that when $\Theta$ in (41) is nonnegative, then inequality (41) gives the refinement of [30] (Theorem 2). By assuming $\gamma, \beta=1$, we obtain the inequality (41) in time scales ([32], Theorem 2.1), when $\phi$ is nonnegative in [32] (Theorem 2.1), then [32] (Theorem 2.1) gives the refinement of [27] (Theorem 3.2). Moreover, the inequality (41) is obtained in fractional calculus [33] by assuming $\mathbb{T}=\mathbb{R}$ and $\gamma, \beta=1$.

Corollary 1. Assume (38)-(40). If $p \geq 2$, then

$$
\begin{aligned}
& \int_{\eta_{1}}^{\eta_{2}} h_{\beta-1}\left(s_{2}, \sigma\left(\vartheta_{1}\right)\right) A_{\kappa}^{p}\left(\phi\left(\vartheta_{2}\right)\right) \zeta\left(\vartheta_{1}\right) \Delta \vartheta_{1} \\
& \quad+\int_{\lambda_{1}}^{\lambda_{2}} h_{\gamma-1}\left(s_{1}, \sigma\left(\vartheta_{2}\right)\right) h_{\beta-1}\left(s_{2}, \sigma\left(\vartheta_{1}\right)\right)\left(\int_{\eta_{1}}^{\eta_{2}}\left|\phi\left(\vartheta_{2}\right)-A_{\kappa}\left(\phi\left(\vartheta_{1}\right)\right)\right|^{p} \frac{\kappa\left(\vartheta_{1}, \vartheta_{2}\right) \zeta\left(\vartheta_{1}\right)}{\chi\left(\vartheta_{1}\right)} \Delta \vartheta_{1}\right) \Delta \vartheta_{2} \\
& \leq \int_{\lambda_{1}}^{\lambda_{2}} h_{\gamma-1}\left(s_{1}, \sigma\left(\vartheta_{2}\right)\right) h_{\beta-1}\left(s_{2}, \sigma\left(\vartheta_{1}\right)\right) \phi^{p}\left(\vartheta_{2}\right) w\left(\vartheta_{2}\right) \Delta \vartheta_{2} .
\end{aligned}
$$

holds for all $\lambda_{\Delta}$-integrable $\phi: \Upsilon_{1} \longrightarrow \mathbb{R}$ such that $\phi\left(\Upsilon_{1}\right) \subset I$.

If $0 \leq p \leq 2$, then (45) holds in reverse direction.

Proof. Use $\Theta(r)=r^{p}$ in Theorem 2 .
Remark 3. We obtain the respective results in time scales ([32], Corollary 2.3) by assuming $\gamma, \beta=1$ in (45).

Remark 4. In particular, if $p=2$ in Corollary 4.2, we get the following identity: 


$$
\begin{aligned}
& \int_{\eta_{1}}^{\eta_{2}} h_{\beta-1}\left(s_{2}, \sigma\left(\vartheta_{1}\right)\right) A_{\mathcal{K}}^{2}\left(\phi\left(\vartheta_{2}\right)\right) \zeta\left(\vartheta_{1}\right) \Delta \vartheta_{1} \\
& \quad+\int_{\lambda_{1}}^{\lambda_{2}} h_{\gamma-1}\left(s_{1}, \sigma\left(\vartheta_{2}\right)\right) h_{\beta-1}\left(s_{2}, \sigma\left(\vartheta_{1}\right)\right)\left(\int_{\eta_{1}}^{\eta_{2}}\left|\phi\left(\vartheta_{2}\right)-A_{\kappa}\left(\phi\left(\vartheta_{1}\right)\right)\right|^{2} \frac{\left(\vartheta_{1}, \vartheta_{2}\right) \zeta\left(\vartheta_{1}\right)}{\chi\left(\vartheta_{1}\right)} \Delta \vartheta_{1}\right) \Delta \vartheta_{2} \\
& =\int_{\lambda_{1}}^{\lambda_{2}} h_{\gamma-1}\left(s_{1}, \sigma\left(\vartheta_{2}\right)\right) h_{\beta-1}\left(s_{2}, \sigma\left(\vartheta_{1}\right)\right) \phi^{2}\left(\vartheta_{2}\right) w\left(\vartheta_{2}\right) \Delta \vartheta_{2} .
\end{aligned}
$$

Corollary 2. Assume (38)-(40), then

$$
\begin{aligned}
& \int_{\eta_{1}}^{\eta_{2}} h_{\beta-1}\left(s_{2}, \sigma\left(\vartheta_{1}\right)\right) e^{A_{\kappa}\left(h\left(\vartheta_{1}\right)\right)} \zeta\left(\vartheta_{1}\right) \Delta \vartheta_{1}+I \\
& \leq \int_{\lambda_{1}}^{\lambda_{2}} h_{\gamma-1}\left(s_{1}, \sigma\left(\vartheta_{2}\right)\right) h_{\beta-1}\left(s_{2}, \sigma\left(\vartheta_{1}\right)\right) h\left(\vartheta_{2}\right) w\left(\vartheta_{2}\right) \Delta \vartheta_{2},
\end{aligned}
$$

holds for all $\lambda_{\Delta}$-integrable $h: \Upsilon_{1} \longrightarrow \mathbb{R}$ such that $\phi\left(\Upsilon_{1}\right) \subset I$ with

$$
\begin{aligned}
A_{\kappa}\left(h\left(\vartheta_{1}\right)\right)= & \frac{1}{\chi\left(\vartheta_{1}\right)}\left(\int_{\lambda_{1}}^{s_{1}} h_{\gamma-1}\left(s_{1}, \sigma\left(\vartheta_{2}\right)\right) \kappa\left(\vartheta_{1}, \vartheta_{2}\right) \ln h\left(\vartheta_{2}\right) \Delta \vartheta_{2}-\int_{\lambda_{2}}^{s_{1}} h_{\gamma-1}\left(s_{1}, \sigma\left(\vartheta_{2}\right)\right) \kappa\left(\vartheta_{1}, \vartheta_{2}\right) \ln h\left(\vartheta_{2}\right) \Delta \vartheta_{2}\right) \\
I= & \int_{\lambda_{1}}^{\lambda_{2}}\left[h_{\gamma-1}\left(s_{1}, \sigma\left(\vartheta_{2}\right)\right) h_{\beta-1}\left(s_{2}, \sigma\left(\vartheta_{1}\right)\right) \times\left(\int_{\eta_{1}}^{\eta_{2}}\left(e^{\left|\ln h\left(\vartheta_{2}\right)-A_{\kappa}\left(h\left(\vartheta_{1}\right)\right)\right|}-\left|\ln h\left(\vartheta_{2}\right)-A_{\kappa}\left(h\left(\vartheta_{1}\right)\right)\right|\right) \frac{\kappa\left(\vartheta_{1}, \vartheta_{2}\right) \zeta\left(\vartheta_{1}\right)}{\chi\left(\vartheta_{1}\right)} \Delta \vartheta_{1}\right)\right] \\
& \Delta \vartheta_{2}+\int_{\lambda_{1}}^{\lambda_{2}} h_{\gamma-1}\left(s_{1}, \sigma\left(\vartheta_{2}\right)\right) h_{\beta-1}\left(s_{2}, \sigma\left(\vartheta_{1}\right)\right) \ln h\left(\vartheta_{2}\right) w\left(\vartheta_{2}\right) \Delta \vartheta_{2}-\int_{\eta_{1}}^{\eta_{2}} h_{\beta-1}\left(s_{2}, \sigma\left(\vartheta_{1}\right)\right)\left(1+A_{\kappa}\left(h\left(\vartheta_{1}\right)\right)\right) \zeta\left(\vartheta_{1}\right) \Delta \vartheta_{1} .
\end{aligned}
$$

Proof. Use $\Theta(r)=e^{r}-r-1$ and $\phi(r)=\ln h(r)$ in Theorem 2.
Remark 5. By choosing $\gamma, \beta=1$ in (47), the respective result is obtained in [32] (Corollary 2.5).

Corollary 3. Assume (38)-(40) and further if $\mathbb{T}=\mathbb{R}$, then

$$
\begin{aligned}
& \int_{\eta_{1}}^{\eta_{2}} \frac{\left(s_{2}-\vartheta_{1}\right)^{\beta-1}}{(\beta-1) !} \Theta\left(\frac{\int_{\lambda_{1}}^{\lambda_{2}}\left(s_{1}-\vartheta_{2}\right)^{\gamma-1} \kappa\left(\vartheta_{1}, \vartheta_{2}\right) \phi\left(\vartheta_{2}\right) \Delta \vartheta_{2}}{\int_{\lambda_{1}}^{\lambda_{2}}\left(\vartheta_{1}-\vartheta_{2}\right)^{\gamma-1} \kappa\left(\vartheta_{1}, \vartheta_{2}\right) \mathrm{d} \vartheta_{2}}\right) \zeta\left(\vartheta_{1}\right) \mathrm{d} \vartheta_{1}
\end{aligned}
$$



$$
\begin{aligned}
& \leq \int_{\lambda_{1}}^{\lambda_{2}} \frac{\left(s_{1}-\vartheta_{2}\right)^{\gamma-1}\left(s_{2}-\vartheta_{1}\right)^{\beta-1}}{(\beta-1) !} \Theta\left(\phi\left(\vartheta_{1}\right)\right)\left(\int_{\eta_{1}}^{\eta_{2}} \frac{\kappa\left(\vartheta_{1}, \vartheta_{2}\right) \zeta\left(\vartheta_{1}\right)}{\int_{\lambda_{1}}^{\lambda_{2}}\left(\vartheta_{1}-\vartheta_{2}\right)^{\gamma-1} \kappa\left(\vartheta_{1}, \vartheta_{2}\right) \mathrm{d} \vartheta_{2}} \mathrm{~d} \vartheta_{1}\right) \mathrm{d} \vartheta_{2} .
\end{aligned}
$$


Proof. If $\mathbb{T}=\mathbb{R}$, then $\sigma\left(\vartheta_{2}\right)=\vartheta_{2}, \forall \vartheta_{2} \in \mathbb{R}$. In this case,

Substituting (50) and (51) in (41), one gets

$$
\begin{aligned}
& h_{\gamma-1}\left(s_{1}, \sigma\left(\vartheta_{2}\right)\right)=\frac{\left(s_{1}-\sigma\left(\vartheta_{2}\right)\right)^{\gamma-1}}{(\gamma-1) !}=\frac{\left(s_{1}-\vartheta_{2}\right)^{\gamma-1}}{(\gamma-1) !}, \\
& h_{\beta-1}\left(s_{2}, \sigma\left(\vartheta_{1}\right)\right)=\frac{\left(s_{2}-\sigma\left(\vartheta_{1}\right)\right)^{\beta-1}}{(\beta-1) !}=\frac{\left(s_{2}-\vartheta_{1}\right)^{\beta-1}}{(\beta-1) !} .
\end{aligned}
$$

$$
\begin{aligned}
& \int_{\eta_{1}}^{\eta_{2}} \frac{\left(s_{2}-\vartheta_{1}\right)^{\beta-1}}{(\beta-1) !} \Theta\left(\frac{\int_{\lambda_{1}}^{\lambda_{2}}\left(s_{1}-\vartheta_{2}\right)^{\gamma-1} \kappa\left(\vartheta_{1}, \vartheta_{2}\right) \phi\left(\vartheta_{2}\right) \Delta \vartheta_{2}}{\int_{\lambda_{1}}^{\lambda_{2}}\left(\vartheta_{1}-\vartheta_{2}\right)^{\gamma-1} \kappa\left(\vartheta_{1}, \vartheta_{2}\right) \mathrm{d} \vartheta_{2}}\right) \zeta\left(\vartheta_{1}\right) \mathrm{d} \vartheta_{1} \\
& +\int_{\lambda_{1}}^{\lambda_{2}}\left(\frac{\left(s_{1}-\vartheta_{2}\right)^{\gamma-1}\left(s_{2}-\vartheta_{1}\right)^{\beta-1}}{(\beta-1) !} \times\left(\begin{array}{c}
\Theta\left(\left|\phi\left(\vartheta_{2}\right)-\frac{\int_{\lambda_{1}}^{\lambda_{2}}\left(s_{1}-\vartheta_{2}\right)^{\gamma-1} \kappa\left(\vartheta_{1}, \vartheta_{2}\right) \phi\left(\vartheta_{2}\right) \Delta \vartheta_{2}}{\int_{\lambda_{1}}^{\lambda_{2}}\left(\vartheta_{1}-\vartheta_{2}\right)^{\gamma-1} \kappa\left(\vartheta_{1}, \vartheta_{2}\right) \mathrm{d} \vartheta_{2}}\right|\right) \\
\eta_{\eta_{1}}^{\eta_{2}}
\end{array}\right) \mathrm{d} \vartheta_{2}\right. \\
& \leq \int_{\lambda_{1}}^{\lambda_{2}} \frac{\left(s_{1}-\vartheta_{2}\right)^{\gamma-1}\left(s_{2}-\vartheta_{1}\right)^{\beta-1}}{(\beta-1) !} \Theta\left(\phi\left(\vartheta_{1}\right)\right)\left(\int_{\eta_{1}}^{\eta_{2}} \frac{\kappa\left(\vartheta_{1}, \vartheta_{2}\right) \zeta\left(\vartheta_{1}\right)}{\int_{\lambda_{1}}^{\lambda_{2}}\left(\vartheta_{1}-\vartheta_{2}\right)^{\gamma-1} \kappa\left(\vartheta_{1}, \vartheta_{2}\right) \mathrm{d} \vartheta_{2}} \mathrm{~d} \vartheta_{1}\right) \mathrm{d} \vartheta_{2} .
\end{aligned}
$$

\section{Inequalities with Special Kernels}

$$
\kappa\left(\vartheta_{1}, \vartheta_{2}\right)= \begin{cases}1, & \text { if } \lambda_{1} \leq \vartheta_{2}<\sigma\left(\vartheta_{1}\right) \leq \lambda_{2} \\ 0, & \text { otherwise }\end{cases}
$$

Corollary 4. Assume (38)-(40) with kernel $\kappa$ such that then

$$
\begin{aligned}
& \int_{\lambda_{1}}^{\lambda_{2}} h_{\beta-1}\left(s_{2}, \sigma\left(\vartheta_{1}\right)\right)\left(\frac{K_{\lambda_{1}}^{\gamma} \phi\left(\sigma\left(\vartheta_{1}\right)\right)}{\mathrm{F}\left(\sigma\left(\vartheta_{1}\right)\right)}\right)^{p} \zeta\left(\vartheta_{1}\right) \Delta \vartheta_{1} \\
& \quad+\int_{\lambda_{1}}^{\lambda_{2}} h_{\gamma-1}\left(s_{1}, \sigma\left(\vartheta_{2}\right)\right) h_{\beta-1}\left(s_{2}, \sigma\left(\vartheta_{1}\right)\right)\left(\int_{\vartheta_{2}}^{\lambda_{2}}\left|\phi\left(\vartheta_{2}\right)-\frac{K_{\lambda_{1}}^{\gamma} \phi\left(\sigma\left(\vartheta_{1}\right)\right)}{F\left(\sigma\left(\vartheta_{1}\right)\right)}\right|^{p} \frac{\zeta\left(\vartheta_{1}\right)}{F\left(\sigma\left(\vartheta_{1}\right)\right)} \Delta \vartheta_{1}\right) \Delta \vartheta_{2} \\
& \leq \int_{\lambda_{1}}^{\lambda_{2}} h_{\gamma-1}\left(s_{1}, \sigma\left(\vartheta_{2}\right)\right) h_{\beta-1}\left(s_{2}, \sigma\left(\vartheta_{1}\right)\right) \phi^{p}\left(\vartheta_{2}\right)\left(\int_{\vartheta_{2}}^{\lambda_{2}} \frac{\zeta\left(\vartheta_{1}\right)}{F\left(\sigma\left(\vartheta_{1}\right)\right)} \Delta \vartheta_{1}\right) \Delta \vartheta_{2},
\end{aligned}
$$

holds for all $\lambda_{\Delta}$-integrable $h: \Upsilon_{1} \longrightarrow \mathbb{R}$ such that $\phi\left(\Upsilon_{1}\right) \subset I . \quad \chi\left(\vartheta_{1}\right)=\int_{\lambda_{1}}^{\sigma\left(\vartheta_{1}\right)} h_{\gamma-1}\left(\sigma\left(\vartheta_{1}\right), \sigma\left(\vartheta_{2}\right)\right) \Delta \vartheta_{2}=F\left(\sigma\left(\vartheta_{1}\right)\right)$,

Proof. By using (53) in (38)-(40), respectively,

$$
w\left(\vartheta_{2}\right)=\int_{\vartheta_{2}}^{\lambda_{2}} \frac{\zeta\left(\vartheta_{1}\right)}{F\left(\sigma\left(\vartheta_{1}\right)\right)} \Delta \vartheta_{1}
$$


$A_{\kappa}\left(\phi\left(\vartheta_{1}\right)\right)=\frac{\int_{\lambda_{1}}^{\sigma\left(\vartheta_{1}\right)} h_{\gamma-1}\left(\sigma\left(\vartheta_{1}\right), \sigma\left(\vartheta_{2}\right)\right) \phi\left(\vartheta_{2}\right) \Delta \vartheta_{2}}{\int_{\lambda_{1}}^{\sigma\left(\vartheta_{1}\right)} h_{\gamma-1}\left(\sigma\left(\vartheta_{1}\right), \sigma\left(\vartheta_{2}\right)\right) \Delta \vartheta_{2}}=\frac{K_{\lambda_{1}}^{\gamma} \phi\left(\sigma\left(\vartheta_{1}\right)\right)}{\mathrm{F}\left(\sigma\left(\vartheta_{1}\right)\right)}$.
We use $\Upsilon_{1}=\Upsilon_{2}=\left[\lambda_{1}, \lambda_{2}\right]$ and (55)-(57) in Corollary 4.2 to get

$$
\begin{aligned}
& \int_{\lambda_{1}}^{\lambda_{2}} h_{\beta-1}\left(s_{2}, \sigma\left(\vartheta_{1}\right)\right)\left(\frac{K_{\lambda_{1}}^{\gamma} \phi\left(\sigma\left(\vartheta_{1}\right)\right)}{\mathrm{F}\left(\sigma\left(\vartheta_{1}\right)\right)}\right)^{p} \zeta\left(\vartheta_{1}\right) \Delta \vartheta_{1} \\
& \quad+\int_{\lambda_{1}}^{\lambda_{2}} h_{\gamma-1}\left(s_{1}, \sigma\left(\vartheta_{2}\right)\right) h_{\beta-1}\left(s_{2}, \sigma\left(\vartheta_{1}\right)\right)\left(\int_{\vartheta_{2}}^{\lambda_{2}}\left|\phi\left(\vartheta_{2}\right)-\frac{K_{\lambda_{1}}^{\gamma} \phi\left(\sigma\left(\vartheta_{1}\right)\right)}{F\left(\sigma\left(\vartheta_{1}\right)\right)}\right|^{p} \frac{\zeta\left(\vartheta_{1}\right)}{F\left(\sigma\left(\vartheta_{1}\right)\right)} \Delta \vartheta_{1}\right) \Delta \vartheta_{2} \\
& \leq \int_{\lambda_{1}}^{\lambda_{2}} h_{\gamma-1}\left(s_{1}, \sigma\left(\vartheta_{2}\right)\right) h_{\beta-1}\left(s_{2}, \sigma\left(\vartheta_{1}\right)\right) \phi^{p}\left(\vartheta_{2}\right)\left(\int_{\vartheta_{2}}^{\lambda_{2}} \frac{\zeta\left(\vartheta_{1}\right)}{F\left(\sigma\left(\vartheta_{1}\right)\right)} \Delta \vartheta_{1}\right) \Delta \vartheta_{2} .
\end{aligned}
$$

Corollary 5. Assume $\Upsilon_{1}=\Upsilon_{2}=\left[\lambda_{1}, \lambda_{2}\right]$ with $\lambda_{2}=\infty$ and (55)-(57) hold, then

$$
\begin{aligned}
& \int_{\lambda_{1}}^{\infty} h_{\beta-1}\left(s_{2}, \sigma\left(\vartheta_{1}\right)\right)\left(\frac{K_{\lambda_{1}}^{\gamma} \phi\left(\sigma\left(\vartheta_{1}\right)\right)}{F\left(\sigma\left(\vartheta_{1}\right)\right)}\right)^{p} \zeta\left(\vartheta_{1}\right) \Delta \vartheta_{1} \\
& \quad+\int_{\lambda_{1}}^{\infty} h_{\gamma-1}\left(s_{1}, \sigma\left(\vartheta_{2}\right)\right) h_{\beta-1}\left(s_{2}, \sigma\left(\vartheta_{1}\right)\right)\left(\int_{\vartheta_{2}}^{\infty}\left|\phi\left(\vartheta_{2}\right)-\frac{K_{\lambda_{1}}^{\gamma} \phi\left(\sigma\left(\vartheta_{1}\right)\right)}{F\left(\sigma\left(\vartheta_{1}\right)\right)}\right|^{p} \frac{\zeta\left(\vartheta_{1}\right)}{F\left(\sigma\left(\vartheta_{1}\right)\right)} \Delta \vartheta_{1}\right) \Delta \vartheta_{2} \\
& \leq \int_{\lambda_{1}}^{\infty} h_{\gamma-1}\left(s_{1}, \sigma\left(\vartheta_{2}\right)\right) h_{\beta-1}\left(s_{2}, \sigma\left(\vartheta_{1}\right)\right) \phi^{p}\left(\vartheta_{2}\right)\left(\int_{\vartheta_{2}}^{\infty} \frac{\zeta\left(\vartheta_{1}\right)}{F\left(\sigma\left(\vartheta_{1}\right)\right)} \Delta \vartheta_{1}\right) \Delta \vartheta_{2},
\end{aligned}
$$

holds for all $\lambda_{\Delta}$-integrable $h: \Upsilon_{1} \longrightarrow \mathbb{R}$ such that $\phi\left(\Upsilon_{1}\right) \subset I$.

Proof. The statement follows from Corollary 4 by using $\lambda_{2}=\infty$.

Remark 6. By assuming $\gamma, \beta=1$ in Corollaries 4 and 5, respectively, we obtain the results given in [32] (Corollaries 3.2 and 3.4) (for $n=1$ ).

\section{Pólya-Knopp Type Inequalities}

Example 3. For $\mathbb{T}=h \mathbb{Z}, \sigma\left(\vartheta_{2}\right)=\vartheta_{2}+h, \forall \vartheta_{2} \in \mathbb{Z}$. In this case,

$$
h_{\gamma-1}\left(s_{1}, \sigma\left(\vartheta_{2}\right)\right)=\frac{\left(s_{1}-\sigma\left(\vartheta_{2}\right)\right)^{\gamma-1}}{(\gamma-1) !}=\frac{\left(s_{1}-\vartheta_{2}-h\right)^{\gamma-1}}{(\gamma-1) !} \text {, }
$$

$$
h_{\beta-1}\left(s_{2}, \sigma\left(\vartheta_{2}\right)\right)=\frac{\left(s_{2}-\sigma\left(\vartheta_{1}\right)\right)^{\beta-1}}{(\beta-1) !}=\frac{\left(s_{2}-\vartheta_{1}-h\right)^{\beta-1}}{(\beta-1) !} \text {. }
$$


By using (60) and (61) in (41), one gets

$$
\begin{aligned}
& \sum_{\vartheta_{1}=\eta_{1}}^{\eta_{2}-1} \frac{\left(s_{2}-\vartheta_{1}-h\right)^{\beta-1}}{(\beta-1) !} \Theta\left(\frac{\sum_{\vartheta_{2}=\lambda_{1}}^{\lambda_{2}-1}\left(s_{1}-\vartheta_{2}-h\right)^{\gamma-1} \kappa\left(\vartheta_{1}, \vartheta_{2}\right) \phi\left(\vartheta_{2}\right)}{\sum_{\vartheta_{2}=\lambda_{1}}^{\vartheta_{1}-1}\left(\vartheta_{1}-\vartheta_{2}-h\right)^{\gamma-1} \kappa\left(\vartheta_{1}, \vartheta_{2}\right)-\sum_{\vartheta_{2}=\lambda_{2}}^{\vartheta_{1}-1}\left(\vartheta_{1}-\vartheta_{2}-h\right)^{\gamma-1} \kappa\left(\vartheta_{1}, \vartheta_{2}\right)}\right) \zeta\left(\vartheta_{1}\right) \\
& \left.+\sum_{\vartheta_{2}=\lambda_{1}}^{\lambda_{2}-1}\left[\left(\begin{array}{c}
\frac{\left(s_{1}-\vartheta_{2}-h\right)^{\gamma-1}\left(s_{2}-\vartheta_{1}-h\right)^{\beta-1}}{(\beta-1) !} \times \\
\sum_{\vartheta_{1}=\eta_{1}}^{\eta_{2}-1} \Theta\left(\phi\left(\vartheta_{2}\right)-\frac{\sum_{\vartheta_{2}=\lambda_{1}}^{\lambda_{2}-1}\left(s_{1}-\vartheta_{2}-h\right)^{\gamma-1} \kappa\left(\vartheta_{1}, \vartheta_{2}\right) \phi\left(\vartheta_{2}\right)}{\sum_{\vartheta_{2}=\lambda_{1}}^{\vartheta_{1}-1}\left(\vartheta_{1}-\vartheta_{2}-h\right)^{\gamma-1} \kappa\left(\vartheta_{1}, \vartheta_{2}\right)-\sum_{\vartheta_{2}=\lambda_{2}}^{\vartheta_{1}-1}\left(\vartheta_{1}-\vartheta_{2}-h\right)^{\gamma-1} \kappa\left(\vartheta_{1}, \vartheta_{2}\right)} \mid\right) \\
\times \frac{\kappa\left(\vartheta_{1}, \vartheta_{2}\right) \zeta\left(\vartheta_{1}\right)}{\left(\sum_{\vartheta_{2}=\lambda_{1}}^{\vartheta_{1}-1}\left(\vartheta_{1}-\vartheta_{2}-h\right)^{\gamma-1} \kappa\left(\vartheta_{1}, \vartheta_{2}\right)-\sum_{\vartheta_{2}=\lambda_{2}}^{\vartheta_{1}-1}\left(\vartheta_{1}-\vartheta_{2}-h\right)^{\gamma-1} \kappa\left(\vartheta_{1}, \vartheta_{2}\right)\right)}
\end{array}\right]\right)\right] \\
& \leq \sum_{\vartheta_{2}=\lambda_{1}}^{\lambda_{2}-1}\left[\begin{array}{c}
\frac{\left(s_{1}-\vartheta_{2}-h\right)^{\gamma-1}\left(s_{2}-\vartheta_{1}-h\right)^{\beta-1}}{(\beta-1) !} \Theta\left(\phi\left(\vartheta_{1}\right)\right) \\
\times \sum_{\vartheta_{1}=\eta_{1}}^{\eta_{2}-1}\left(\frac{\kappa\left(\vartheta_{1}, \vartheta_{2}\right) \zeta\left(\vartheta_{1}\right)}{\left(\sum_{\vartheta_{2}=\lambda_{1}}^{\vartheta_{1}-1}\left(\vartheta_{1}-\vartheta_{2}-h\right)^{\gamma-1} \kappa\left(\vartheta_{1}, \vartheta_{2}\right)-\sum_{\vartheta_{2}=\lambda_{2}}^{\vartheta_{1}-1}\left(\vartheta_{1}-\vartheta_{2}-h\right)^{\gamma-1} \kappa\left(\vartheta_{1}, \vartheta_{2}\right)\right)}\right)
\end{array}\right] .
\end{aligned}
$$


$\Upsilon_{1}=\Upsilon_{2}=\left[\lambda_{1}, \infty\right) \cap \mathbb{T}, \quad \lambda_{1} \geq 0$, and $\zeta: \Upsilon_{2} \longrightarrow \mathbb{R}_{+}$is a $\mu_{\Delta}$-integrable function. For $p \geq 2$ inequality,

$$
\begin{aligned}
& \sum_{\vartheta_{1} \in\left[\lambda_{1}, \infty\right)_{\mathbb{T}}} \frac{\left(s_{2}-\vartheta_{1}-1\right)^{\beta-1} \zeta\left(\vartheta_{1}\right)}{(\beta-1) !}\left(\frac{\sum_{\vartheta_{2} \in\left[\lambda_{1}, \sigma\left(\vartheta_{1}\right)\right)_{\mathbb{T}}}\left(\vartheta_{1}-\vartheta_{2}\right)^{\gamma-1} \phi\left(\vartheta_{2}\right) \mu\left(\vartheta_{2}\right)}{\sum_{\vartheta_{2} \in\left[\lambda_{1}, \sigma\left(\vartheta_{1}\right)\right)_{\mathbb{T}}}\left(\vartheta_{1}-\vartheta_{2}\right)^{\gamma-1} \mu\left(\vartheta_{2}\right)}\right)^{p} \mu\left(\vartheta_{1}\right)
\end{aligned}
$$

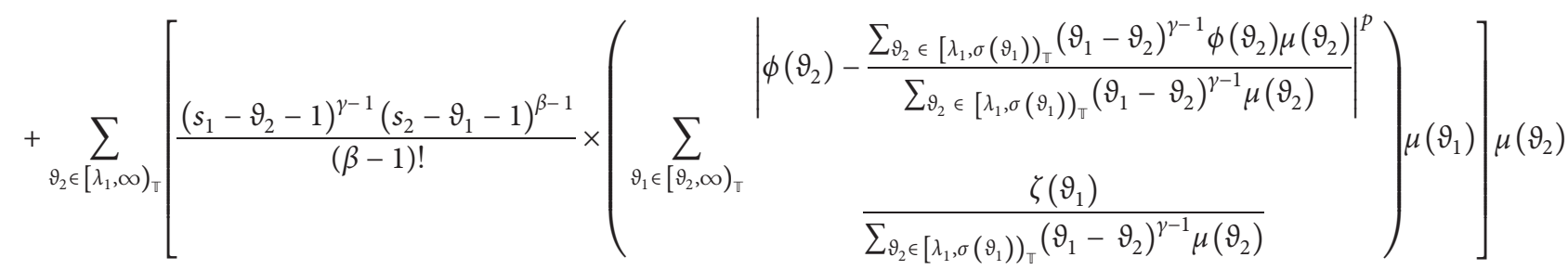

$$
\begin{aligned}
& \leq \sum_{\vartheta_{2} \in\left[\lambda_{1}, \infty\right)_{\mathbb{T}}} \frac{\left(s_{1}-\vartheta_{2}-1\right)^{\gamma-1}\left(s_{2}-\vartheta_{1}-1\right)^{\beta-1} \phi^{p}\left(\vartheta_{2}\right)}{(\beta-1) !}\left(\sum_{\vartheta_{1} \in\left[\vartheta_{2}, \infty\right)_{\mathbb{T}}} \frac{\zeta\left(\vartheta_{1}\right)}{\sum_{\vartheta_{2} \in\left[\lambda_{1}, \sigma\left(\vartheta_{1}\right)\right)_{\mathbb{T}}}\left(\vartheta_{1}-\vartheta_{2}\right)^{\gamma-1} \mu\left(\vartheta_{2}\right)} \mu\left(\vartheta_{1}\right)\right) \mu\left(\vartheta_{2}\right),
\end{aligned}
$$

holds for all $\lambda_{\Delta}$-integrable $\phi: \Upsilon_{1} \longrightarrow \mathbb{R}$ such that $\phi\left(\Upsilon_{1}\right) \subset I, \quad$ Proof. If $\mathbb{T}$ consists of isolated points, then where $\mu(r)=\sigma(r)-r$. 


$$
\begin{aligned}
h_{\gamma-1}\left(s_{1}, \sigma\left(\vartheta_{2}\right)\right) & =\frac{\left(s_{1}-\sigma\left(\vartheta_{2}\right)\right)^{\gamma-1}}{(\gamma-1) !}=\frac{\left(s_{1}-\vartheta_{2}-1\right)^{\gamma-1}}{(\gamma-1) !}, \\
h_{\gamma-1}\left(\sigma\left(\vartheta_{1}\right), \sigma\left(\vartheta_{2}\right)\right) & =\frac{\left(\sigma\left(\vartheta_{1}\right)-\sigma\left(\vartheta_{2}\right)\right)^{\gamma-1}}{(\gamma-1) !}=\frac{\left(\vartheta_{1}-\vartheta_{2}\right)^{\gamma-1}}{(\gamma-1) !}, \\
h_{\beta-1}\left(s_{2}, \sigma\left(\vartheta_{1}\right)\right) & =\frac{\left(s_{2}-\sigma\left(\vartheta_{1}\right)\right)^{\beta-1}}{(\beta-1) !}=\frac{\left(s_{2}-\vartheta_{1}-1\right)^{\beta-1}}{(\beta-1) !} .
\end{aligned}
$$

By using (64)-(66) in Corollary 5, one gets

$$
\begin{aligned}
& \sum_{\vartheta_{1} \in\left[\lambda_{1}, \infty\right)_{\top}} \frac{\left(s_{2}-\vartheta_{1}-1\right)^{\beta-1} \zeta\left(\vartheta_{1}\right)}{\beta-1 !}\left(\frac{\sum_{\vartheta_{2} \in\left[\lambda_{1}, \sigma\left(\vartheta_{1}\right)\right)_{\mathbb{T}}}\left(\vartheta_{1}-\vartheta_{2}\right)^{\gamma-1} \phi\left(\vartheta_{2}\right) \mu\left(\vartheta_{2}\right)}{\sum_{\vartheta_{2} \in\left[\lambda_{1}, \sigma\left(\vartheta_{1}\right)\right)_{\top}}\left(\vartheta_{1}-\vartheta_{2}\right)^{\gamma-1} \mu\left(\vartheta_{2}\right)}\right) \mu\left(\vartheta_{1}\right)
\end{aligned}
$$

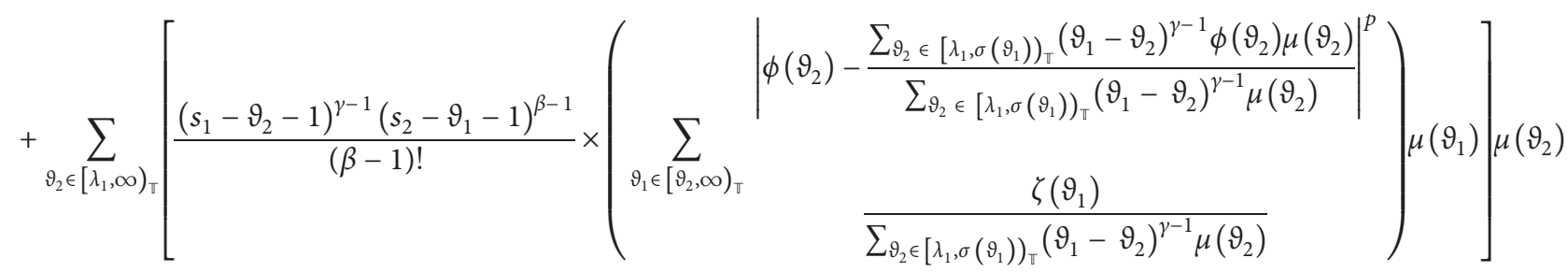

$$
\begin{aligned}
& \leq \sum_{\left.\vartheta_{2} \in\left[\lambda_{1}, \infty\right)\right)_{\top}} \frac{\left(s_{1}-\vartheta_{2}-1\right)^{\gamma-1}\left(s_{2}-\vartheta_{1}-1\right)^{\beta-1} \phi^{p}\left(\vartheta_{2}\right)}{(\beta-1) !}\left(\sum_{\vartheta_{1} \in\left[\vartheta_{2}, \infty\right)_{\top}} \frac{\zeta\left(\vartheta_{1}\right)}{\sum_{\vartheta_{2} \in\left[\lambda_{1}, \sigma\left(\vartheta_{1}\right)\right)_{\top}}\left(\vartheta_{1}-\vartheta_{2}\right)^{\gamma-1} \mu\left(\vartheta_{2}\right)} \mu\left(\vartheta_{1}\right)\right) \mu\left(\vartheta_{2}\right) \text {. }
\end{aligned}
$$

Remark 7. By choosing $\gamma, \beta=1$ in (63), the respective Polya-Knopp type inequality is obtained in time scales ([32], Corollary 4.1).
Example 4. If we consider $\mathbb{T}=h \mathbb{N}=\{h \iota: \iota \in \mathbb{N}\}$ with fixed $h>0, \lambda_{1}=h$ and taking $\zeta\left(\vartheta_{1}\right)=\left(1 / \sigma\left(\vartheta_{1}\right)\right)$. (63) takes the form,

$$
\begin{aligned}
& \sum_{\iota=1}^{\infty} \frac{(\mathscr{J}-\iota-1)^{\beta-1}}{(\beta-1) !(\iota+1)}\left(\frac{\sum_{\kappa=1}^{\iota}(\iota-\kappa)^{\gamma-1} \phi(\kappa(h))}{\sum_{\kappa=1}^{\iota}(\iota-\kappa)^{\gamma-1}}\right)^{p} \\
& \quad+\sum_{\kappa=1}^{\infty}\left[\frac{(\mathrm{lh}-\kappa(\mathrm{h})-\mathrm{h})^{\gamma-1}(\mathscr{J} h-\iota h-h)^{\beta-1}}{(\beta-1) !} \times\left(\sum_{\iota=1}^{\infty}\left|\phi\left(\vartheta_{2}\right)-\frac{\sum_{\kappa=1}^{\iota}(\iota-\kappa)^{\gamma-1} \phi\left(\vartheta_{2}\right)}{\sum_{\kappa=1}^{l}(\iota-\kappa)^{\gamma-1}}\right|^{p} \frac{1}{\iota+1 \sum_{\kappa=1}^{\iota}(\iota h-\kappa(h))^{\gamma-1}}\right)\right] \\
& \leq \sum_{\kappa=1}^{\infty} \frac{(\mathrm{lh}-\kappa(\mathrm{h})-\mathrm{h})^{\gamma-1}(\mathscr{J}-\iota-1)^{\beta-1} \phi(\kappa(h))}{(\beta-1) !}\left(\sum_{l=\kappa}^{\infty} \frac{1}{\iota+1 \sum_{\kappa=1}^{l}(\iota h-\kappa(h))^{\gamma-1}}\right) .
\end{aligned}
$$

Remark 8. Notice that (68) becomes [32] Example 4.3 by choosing $\gamma, \beta=1$.
Example 5. If we consider time scale $\mathbb{T}=\mathbb{N}^{2}=\left\{\iota^{2}: \iota \in \mathbb{N}\right\}$, $\lambda_{1}=1$ and

$$
\zeta\left(\vartheta_{1}\right)=\frac{2\left(\sigma\left(\vartheta_{1}\right)-1\right)}{\left(\sigma\left(\vartheta_{1}\right)-\vartheta_{1}\right)\left(2 \sqrt{\vartheta_{1}}+3\right)}=\frac{2\left((\iota+1)^{2}-1\right)}{\left((\iota+1)^{2}-\iota^{2}\right)(2 \iota+3)}=\frac{2 \iota(\iota+2)}{(2 \iota+1)(2 \iota+3)} .
$$


(63) takes the form,

$$
\begin{aligned}
& \sum_{\iota=1}^{\infty} \frac{\left(\mathcal{F}^{2}-(\iota+1)^{2}\right)^{\beta-1} 2 \iota(\iota+2)}{(\beta-1) !(2 \iota+3)}\left(\frac{\sum_{\kappa=1}^{\iota}\left((\iota+1)^{2}-(\kappa+1)^{2}\right)^{\gamma-1} \phi\left(\kappa^{2}\right)(2 \kappa+1)}{\sum_{\kappa=1}^{\iota}\left((\iota+1)^{2}-(\kappa+1)^{2}\right)^{\gamma-1}(2 \kappa+1)}\right)^{p} \\
& +\sum_{\kappa=1}^{\infty}\left[\frac{\left(1^{2}-(\kappa+1)^{2}\right)^{\gamma-1}\left(\mathcal{F}^{2}-(\iota+1)^{2}\right)^{\beta-1}}{(\beta-1) !} \times\left(\begin{array}{c}
\sum_{\iota=\kappa}^{\infty} \\
\times \frac{\phi\left(\kappa^{2}\right)-\frac{\sum_{\kappa=1}^{\iota}\left((\iota+1)^{2}-(\kappa+1)^{2}\right)^{\gamma-1} \phi\left(\kappa^{2}\right)(2 \kappa+1)}{\sum_{\kappa=1}^{\iota}\left((\iota+1)^{2}-(\kappa+1)^{2}\right)^{\gamma-1}(2 \kappa+1)} \mid}{(2 \iota+3) \sum_{\kappa=1}^{\iota}\left((\iota+1)^{2}-(\kappa+1)^{2}\right)^{\gamma-1}(2 \kappa+1)}
\end{array}\right)\right](2 \kappa+1) \\
& \leq \sum_{\kappa=1}^{\infty}\left[\frac{\left(1^{2}-k h-h\right)^{\gamma-1}\left(\mathscr{g}^{2}-n h-h\right)^{\beta-1} \phi\left(\kappa^{2}\right)}{(\beta-1) !} \times\left(\sum_{l=\kappa}^{\infty} \frac{2 \iota(\iota+2)}{(2 \iota+3) \sum_{\kappa=1}^{\iota}\left((\iota+1)^{2}-(\kappa+1)^{2}\right)^{\gamma-1}(2 \kappa+1)}\right)\right](2 \kappa+1) .
\end{aligned}
$$

Example 6. If $\mathbb{T}=q^{\mathbb{N}}=\left\{q^{n}: n \in \mathbb{N}\right\}$ with fixed $q>1$, then $\sigma(\vartheta)=q \vartheta=q^{n+1}$, and assume

$$
\begin{aligned}
& h_{\gamma-1}\left(s_{1}, \sigma\left(\vartheta_{2}\right)\right)=\prod_{v=0}^{\gamma-2} \frac{s_{1}-q^{v} \sigma\left(\vartheta_{2}\right)}{\sum_{\mu=0}^{v} q^{\mu}}=\prod_{v=0}^{\gamma-2} \frac{s_{1}-q^{v+k+1}}{\sum_{\mu=0}^{v} q^{\mu}}, \\
& h_{\beta-1}\left(s_{2}, \sigma\left(\vartheta_{1}\right)\right)=\prod_{v=0}^{\beta-2} \frac{s_{2}-q^{v} \sigma\left(\vartheta_{1}\right)}{\sum_{\mu=0}^{v} q^{\mu}}=\prod_{v=0}^{\beta-2} \frac{s_{2}-q^{v+n+1}}{\sum_{\mu=0}^{v} q^{\mu}} .
\end{aligned}
$$

In this case,

By using (63), (71)-(73) take the form,

$$
\begin{aligned}
& \sum_{n=1}^{\infty}\left(\left(\prod_{v=0}^{\beta-2} \frac{s_{2}-q^{v+n+1}}{\sum_{\mu=0}^{v} q^{\mu}}\right) \frac{2\left(q^{n+1}-1\right)}{q^{n}\left(2 q^{n}+3\right)}\left(\frac{\sum_{k=1}^{n}\left(\prod_{v=0}^{\gamma-2}\left(s_{1}-q^{v+k+1} / \sum_{\mu=0}^{v} q^{\mu}\right)\right) \phi\left(q^{k}\right)}{\sum_{k=1}^{n}\left(\prod_{v=0}^{\gamma-2}\left(q^{n}-q^{v+k+1} / \sum_{\mu=0}^{v} q^{\mu}\right)\right) \phi\left(q^{k}\right)}\right)^{p}\right) \\
& \quad+\sum_{k=1}^{\infty}\left(\prod_{v=0}^{\gamma-2} \frac{s_{1}-q^{v+k+1}}{\sum_{\mu=0}^{v} q^{\mu}} \cdot \prod_{v=0}^{\beta-2} \frac{s_{2}-q^{v+n+1}}{\sum_{\mu=0}^{v} q^{\mu}} \sum_{n=k}^{\infty}\left(\left|\phi\left(q^{k}\right)-\frac{\sum_{k=1}^{n}\left(\prod_{v=0}^{\gamma-2}\left(s_{1}-q^{v+k+1} / \sum_{\mu=0}^{v} q^{\mu}\right)\right) \phi\left(q^{k}\right)}{\sum_{k=1}^{n}\left(\prod_{v=0}^{\gamma-2}\left(q^{n}-q^{v+k+1} / \sum_{\mu=0}^{v} q^{\mu}\right)\right)}\right|^{p} \frac{\left(2\left(q^{n+1}-1\right) / q^{n}\left(2 q^{n}+3\right)\right)}{\sum_{k=1}^{n} \prod_{v=0}^{\gamma-2}\left(q^{n}-q^{v+k+1} / \sum_{\mu=0}^{v} q^{\mu}\right)}\right)\right) \\
& \leq \sum_{k=1}^{\infty}\left(\prod_{v=0}^{\gamma-2} \frac{s_{1}-q^{v+k+1}}{\sum_{\mu=0}^{v} q^{\mu}} \cdot \prod_{v=0}^{\beta-2} \frac{s_{2}-q^{v+n+1}}{\sum_{\mu=0}^{v} q^{\mu}}\left(\phi\left(q^{k}\right)\right)^{p} \sum_{n=k}^{\infty}\left(\frac{\left(2\left(q^{n+1}-1\right) / q^{n}\left(2 q^{n}+3\right)\right)}{\sum_{k=1}^{n}\left(\prod_{v=0}^{\gamma-2}\left(q^{n}-q^{v+k+1} / \sum_{\mu=0}^{v} q^{\mu}\right)\right)}\right)\right) .
\end{aligned}
$$

Remark 9. By choosing $\gamma, \beta=1$ in (70), the respective Polya-Knopp type inequality is obtained in time scales ([32], Example 4.4).

\section{Conclusion}

In the paper, some dynamic inequalities of Jensen and Hardy for superquadratic functions with Riemann-Liouville delta fractional integrals are generalized. Particular cases of obtained inequalities have been given in time scales [32] by assuming $\gamma, \beta=1$ and respective inequalities in fractional calculus [33] by choosing $\mathbb{T}=\mathbb{R}$ are also obtained. Furthermore, some discrete fractional inequalities for superquadratic functions are obtained by choosing $\mathbb{T}=h \mathbb{Z}$ and $\mathbb{T}=\mathbb{N}^{2}$. Inequalities in quantum discrete calculus are also obtained by choosing $\mathbb{T}=q^{\mathbb{Z}}$, which are new, up to the knowledge of authors.

\section{Data Availability}

All data generated or analyzed during this study are included in this published article. There are no experimental data in this article.

\section{Conflicts of Interest}

The authors declare that they have no conflicts of interest. 


\section{References}

[1] J. Dolbeault, M. J. Esteban, and E. Séré, "On the eigenvalues of operators with gaps. Application to Dirac operators," Journal of Functional Analysis, vol. 174, no. 1, pp. 208-226, 2000.

[2] P. Mironescu, "The role of the Hardy type inequalities in the theory of function spaces," Revue roumaine de mathématiques pures et appliquées, vol. 63, no. 4, pp. 447-525, 2018.

[3] G. H. Hardy, J. E. Littlewood, and G. Pólya, Inequalities, Cambridge University Press, Cambridge, UK, 1988.

[4] E. K. Godunova, "Inequalities based on convex functions," Izvestiya Vysshikh Uchebnykh Zavedenii. Matematika, vol. 47, pp. 45-53, 1965.

[5] S. Kaijser, L. Nikolova, L.-E. Persson, and A. Wedestig, "Hardy-type inequalities via convexity," Mathematical Inequalities \& Applications, vol. 8, no. 3, pp. 403-417, 2005.

[6] G. H. Hardy, "Note on a theorem of Hilbert," Mathematische Zeitschrift, vol. 6, no. 3-4, pp. 314-317, 1920.

[7] G. H. Hardy, "Notes on some points in the integral calculus (LX). An inequality between integrals," Messenger Math, vol. 54, pp. 150-156, 1925.

[8] G. H. Hardy, "Notes on some points in the integral calculus (LXIT)," Messenger of Math, vol. 57, pp. 12-16, 1928.

[9] J. E. Littlewood and G. H. Hardy, "Elementary theorems concerning power series with positive coefficients and moment constants of positive functions," Journal für die reine und angewandte Mathematik, vol. 1927, no. 157, pp. 141-158, 2009.

[10] B. G. Pachpatte, "A note on Copson's inequality involving series of positive terms," Tamkang Journal of Mathematics, vol. 21, no. 6, pp. 13-19, 1990.

[11] S. H. Saker, R. R. Mahmoud, M. M. Osman, and R. P. Agarwal, "Some new generalized forms of Hardy's type inequality on time scales," Journal of Mathematical Analysis and Applications, vol. 20, no. 2, pp. 459-481, 2017.

[12] S. H. Saker and D. O'Regan, "Hardy and littlewood inequalities on time scales," Bulletin of the Malaysian Mathematical Sciences Society, vol. 39, no. 2, pp. 527-543, 2016.

[13] B. Opic and A. Kufner, Hardy-Type Inequalities, Pitman Research Notes in Mathematics Series, Longman Scientific and Technical, Harlow, UK, 1990.

[14] S. Abramovich, J. Baric, and J. Pecaric, "A variant of Jessen's inequality of Mercer's type for superquadratic functions," Journal of Inequalities in Pure and Applied Mathematics, vol. 9 , no. 3, p. 13, 2008.

[15] S. Banic and S. Varosanec, "Functional inequalities for superquadratic functions," International Journal of Pure and Applied Mathematics, vol. 43, no. 4, p. 537, 2008.

[16] D. T. Nguyen, N. Lam-Hoang, and T. A. Nguyen, "Hardy and Rellich inequalities with exact missing terms on homogeneous groups," Journal of the Mathematical Society of Japan, vol. 71, no. 4, pp. 1243-1256, 2019.

[17] X. Cabre and P. Miraglio, "Universal Hardy-Sobolev inequalities on hypersurfaces of Euclidean space," 2019, https:// arxiv.org/abs/1912.09282.

[18] N. T. Duy, N. Lam, N. Triet, and W. Yin, "Improved Hardy inequalities with exact remainder terms," Mathematical Inequalities \& Applications, vol. 23, no. 4, pp. 1205-1226, 2020.

[19] N. Kutev and T. Rangelov, "Hardy inequalities with double singular weights," 2020, https://arxiv.org/abs/2001.07368.

[20] A. Salort, "Hardy inequalities in fractional Orlicz-Sobolev spaces," 2020, https://arxiv.org/abs/2009.06431.

[21] L. Akin, "A new approach for the fractional integral operator in time scales with variable exponent Lebesgue spaces," Fractal Fract, vol. 5, no. 1, p. 7, 2021.
[22] L. Akin, "On innovations of $n$-dimensional integral-type inequality on time scales," Advances in Difference Equations, vol. 2021, p. 148, 2021.

[23] S. G. Samko, Fractional Integrals and Derivatives, Theory and Applications, Nauka I Tekhnika, Minsk, Belarus, 1987.

[24] E. T. Whittaker and G. N. Watson, A Course in Modern Analysis, Cambridge University Press, Cambridge, UK, 1927.

[25] M. Bohner and A. Peterson, Dynamic Equations on Time Scales: An Introduction with Applications, Springer Science \& Business Media, Berlin, Germany, 2001.

[26] G. A. Anastassiou, "Principles of delta fractional calculus on time scales and inequalities," Mathematical and Computer Modelling, vol. 52, no. 3-4, pp. 556-566, 2010.

[27] M. J. Bohner, A. Nosheen, J. Pečarić, and A. Younus, "Some dynamic Hardy-type inequalities with general kernel," Journal of Mathematical Inequalities, vol. 8, no. 1, pp. 185-199, 2014.

[28] B. Karpuz, "Unbounded oscillation of higher-order nonlinear delay dynamic equations of neutral type with oscillating coefficients," Electronic Journal of Qualitative Theory of Differential Equations [electronic Only], vol. 34, pp. 1-14, 2009.

[29] S. Abramovich, G. Jameson, and G. Sinnamon, "Refining Jensen's inequality," Bulletin Mathématique De La Société Des Sciences Mathématiques De Roumanie, vol. 47, pp. 3-14, 2004.

[30] A. Nosheen, U. Hanif, K. A. Khan, and H. Ahmed, Some Hardy-Type Inequalities via Delta Fractional Integrals, Elsevier, Amsterdam, Netherlands, 2020.

[31] J. Baric, R. Bibi, M. Bohner, and J. Pecaric, "Time scales integral inequalities for superquadratic functions," Journal of the Korean Mathematical Society, vol. 50, no. 3, pp. 465-477, 2013.

[32] J. Barić, A. Nosheen, and J. Pečarić, "Time scale Hardy-type inequalities with general kernel for superquadratic functions," Annals of Functional Analysis, vol. 5, pp. 61-73, 2014.

[33] S. Abramovich, K. Krulić, J. Pečarić, and L. E. Persson, "Some new refined Hardy type inequalities with general kernels and measures," Aequationes Mathematicae, vol. 79, no. 1-2, pp. 157-172, 2010. 